\title{
Application of neural networks in cardiovascular decision support systems
}

\author{
Konnova N.S., Basarab M.A.
}

\begin{abstract}
The development of medical decision support systems, especially in the field of cardiology, is an important and urgent task. The report considers the main requirements for the recognition system used to assess the functional state of the cardiovascular system (CVS). The description of the general scheme of the developed decision support system based on the identification and classification of CVS states is given. As various types of neural networks and other classifiers based on machine learning are often used in problems of the cardiovascular states identification, here, the results obtained with the help of different architectures of such classifiers are examined. A proprietary numerical experiment with real clinical data (confirmed by crossvalidation) is performed on the basis of the most efficient neural networks with refinements for generalization and data preprocessing.
\end{abstract}

Keywords-machine learning, deep neural networks, diagnostics of the cardiovascular system state, decision support system, digital signal processing, system analysis

\section{Introduction. Statement of the problem}

Diagnosis of the cardiovascular system state of a person is one of the most important tasks of cardiology. Known methods of diagnosing the cardiovascular system (ultrasound diagnostics, radionuclide methods, radiocontrast studies, etc.) are aimed at revealing various pathologies, as well as a number of factors that cause not only a "mechanical" anomaly in the structure of the vascular system and the heart muscle, but also a violation of physiological rhythms of their work. Most methods can be classified into the following groups: active methods that presuppose effects on the body of fields and substances (angiography, Doppler echocardiography, electrolethelymography, etc.), and methods of passive diagnostics based on the registration of physical fields generated by the body itself (ECG, ICG, PCG, etc.) [1].

Konnova Natalia Sergeevna, Ph.D.

Bauman Moscow State Technical University

Russia

Basarab Mikhail Alekseevich, Prof.

Bauman Moscow State Technical University

Russia

The reported study was funded by RFBR according to the research project № 18-29-02019.

Data collection was financed according to project № 40393 of the

Fund for the Promotion of Innovations of the Russian Federation.
These methods have different informativeness, various advantages and disadvantages. However, the choice of a method for recording cardiovascular performance is not the only and probably not the most important task in the automation of cardiac diagnostics. The problem of studying the human blood circulation as a complex biomechanical system has not been completely solved. First of all, this is due to the lack of quality models for predicting CVS diseases and the presence of a number of difficulties and limitations in diagnosing heart and vascular diseases. So, in view of the need to identify the peculiarities of central and regional hemodynamics, including intraoperatively supporting decision-making in cardiac surgery, research and application of new mathematical methods and algorithms for assessing the basic quantitative and qualitative indices of such physiological signals, analysis of their internal organization and structure have great practical and theoretical interest. In this connection, the problems of analyzing these complex biological systems with their features taken into account, including the presence of nonlinear components and a plurality of cycles in the dynamics of their fluctuations, from the standpoint of methods of system and mathematical analysis become relevant.

\section{Development of decision support systems in cardiology from the standpoint of system analysis}

\section{A. Structure of tasks and analysis of requirements for decision support systems in cardiology}

Now then, the object of research is a cardiovascular system consisting of a heart - a biopump - and blood vessels - hollow tubes of various calibres. Regulation of blood circulation in CVS is complex, and the behavior of this system is difficult to formalize. Nevertheless, it is required to carry out the identification of CVS states according to the parameters of the system accessible to the researcher. For this purpose, we propose to use, for the CVS presentation, a time series model constructed from electrocardiography (ECG) data, ultrasonic Doppler blood flow velocity sensors of various nature (peripheral blood flow, coronary blood flow, etc.) (flowmetry) and seismocardiography (SCG).

The subject of the study is the identification of the practical applicability of machine learning methods based on the use of neural networks to automate the diagnosis of the 
CVS state. The objectives of this research also include issues of preliminary preparation and processing of data, their optimization for use in expert systems.

It should also be remembered that the development of microelectronics and telecommunications allows the patient to be included in the single information space of the healthcare system, regardless of his location. This can be done, for example, by recording biosignals of the cardiovascular system with the help of sensors, and the subsequent transfer of these biosignals through communication channels to medical monitoring and information processing centers, where a virtual physiological image of the patient is created through mathematical models that describes human physiological activity including cardiovascular subsystem. This is especially true for large countries, where the geographical remoteness of the patient from the nearest cardiological center can be very significant, which makes it difficult to observe him. Therefore, the process of modernizing the health system is accompanied by the active introduction of information and communication technologies, the creation of virtual infrastructures for the development of telemedicine services: the functioning of electronic registries, the provision of access to electronic medical records of patients, etc. [2]. Thus, when developing decision support systems in cardiology, it is highly desirable, among other things (universality, portability, etc.) to take into account the application of telemedicine technologies.

The analysis of the works devoted to the solution of the problem of provision of remote and automated assistance to cardiac patients shows that the researches in this subject area are conducted in the following main directions [3]:

- development of software complexes and tools for support systems for making diagnostic decisions for specific cardiovascular pathologies;

- development of methods for constructing decision rules to predict various types of cardiovascular diseases;

- $\quad$ search for methods for determining informative points and patterns as additional indicators for predicting and diagnosing cardiovascular pathologies;

- development of methods for mathematical modeling and reconstruction of complex objects, including the restoration of the three-dimensional shape of the heart and vessels for the purpose of its software visualization;

- development of methods and algorithmic tools for constructing and simulating the functioning of a chaotic machine for creating a virtual model of the cardiovascular system of a person in medical diagnostics.

Due to the poor structuredness of the cardiologist's tasks, because of the lack of clear signs of assessing the state of the cardiovascular system, solutions based on the use of the cardiologist's intelligence as an expert system are usually suggested. However, the rapid growth in the amount of information that physicians need in their practical activities in the diagnosis and treatment of diseases often leads to the fact that specialists in decision-making come from previous professional experience and their own intuition, and not from an analysis of objective data. In such a situation, it is difficult to avoid medical errors, the social and economic importance of which is extremely high. There is a need to increase the number of interdependent indicators of the organism's activity taken into account and analyzed by the physician, which further complicates the task of the cardiologist. Therefore, as never before, the problem of reducing ill-conceived decisions in medicine is topical and its solution lies in the use of computer diagnostics based on artificial intelligence, trained on real clinical data under the supervision of qualified experts.

The use of machine learning methods based on artificial intelligence, namely artificial neural networks, has recently shown its effectiveness and has found a successful application in the development of automation of medical activities, including in the field of cardiology. Appropriate developments are being carried out by well-known international companies, such as, for example, Google [4] and ZebraMed. These developments show the first successful steps in clinical trials and prove the practical effectiveness of using neural network methods in automation diagnostics tasks, but the range of tasks and requirements for such systems is very wide and the problems of developing universal expert systems are far from complete. In addition, while such developments are focused on solving highly specialized problems, which do not include the task of considering in this study.

\section{B. Model of the recognition system used to assess the functional status of the CVS}

So, automated diagnosis of human cardiovascular system diseases involves the registration of physiological signals that carry information about the state of the system. As mentioned above, this paper suggests the use of ECG series, Doppler flowmetry and seismic cardiograms as such physiological signals. Thus, the researcher gets at his disposal a very diverse data on the analyzed system. These data include the main types of recorded CVS indicators, by which deviations can be noticed and the state diagnosed. The results of the investigation and processing of these signals [5, 6] indicate that these signals contain very accurate and reliable information about the CVS for its use in the formation of a decision support system.

To automate the diagnosis of CVS diseases, it is necessary to build a recognition system based on a comprehensive mathematical analysis of these signals. Hence the need to process the corresponding time series. In this case, the main purpose of mathematical analysis of time series is their classification. If found classes fully correspond to those functional states in which the CVS can be, then the problem of constructing the recognition system will be completely solved. In general, the recognition system represents a system of decision rules that are based on various properties of the analyzed time series. However, as mentioned above, due to the 
poor structuredness of the tasks solved by the cardiologist and the absence of clear signs of assessing the status of the CVS, the development of methods for constructing decision rules for predicting various types of diseases of the cardiovascular system is extremely difficult. Essentially, we are talking about the construction of a recognition system, which allows, with an acceptable accuracy for medical practice, to relate the test subjects to one of the predefined classes. In the first approximation, the absolute majority of works in this area, e.g. $[8,9]$, consider the binary classification of subjects: the first class is the class of practically healthy subjects; the second class is the class of people who have any particular pathology being studied. First of all, this is because the problem of splitting input objects by methods of machine learning into two classes is solved most simply, in a known way. When using neural networks for this problem, only one additional hidden layer is enough, and generally speaking, no matter how the phase space of states of the system in the plan of geometrical interpretation actually looks for the case under consideration, it is possible mathematically to translate such a space into a space where the objects will be divided into two class only by a hyperplane (see Fig. 1).

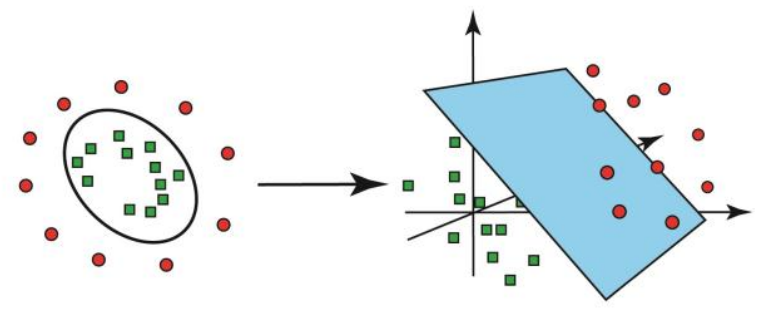

Figure 1. Example of transferring data to another space where data can be separated.

A more precise classification requires the introduction of separate classes for people with the contemplated disease, for people who are sick with disease associated with the contemplated disease, as well as for people who have a certain pathology, but this pathology is not directly caused by disturbances in the work of the CVS. This is often found in cardiology, if we analyze the patient's clinical records, we will see that in the field of cardiology the patient suffers seldom from a single disease. As a rule, there are several associated diseases. For example: [Critical valvular stenosis of the aortic estuary. Calcinosis III st. Hemodynamic angina. Presyncopal states. Paroxysmal atrial fibrillation. Hypertensive disease of III stage, III degree with heart damage...]. For practical application it would be important to highlight the signs of a key disease. Moreover, people who have one or another pathology (not necessarily related to CVS) can be, for example, as a result of taking medications or any other compensatory mechanisms, in a changed functional state. This further complicates the classification of the subjects, since it is necessary to introduce separate classes for both a "pure" functional state, corresponding to a situation with a clearly observed deviation of the state from the norm, and for the "changed" functional state. For each permissible classification of the test subjects, a different recognition system can be built, herewith, when the hierarchical classification is used, the recognition scheme will have the form of a sequence of stages, each of which examines the subjects of certain classes. Then you can choose the number of recognition steps, select specific classes for each stage, and use its own recognition quality criteria at each stage.

\section{Embodiments of the recognition and classification system based on the method of analyzing the CVS signals}

Since at our disposal there are at least three different types of signals (options for recording the activity of the cardiovascular system: flowmetry, ECG, seismic cardiography), each of these types can have its own recognition system, and it is possible to choose from three recognition systems that one, which is more effective. A comparative analysis of the recognition results obtained for each type of signals can give additional information about the subjects, but if signals of two or more kinds (in this case, three types of signals) are considered, it should be borne in mind that these signals have completely different properties, and therefore, they should not be considered as homogeneous data in the construction of decision rules.

If we consider these three recognition systems as independent, then we can build a system of decision rules of a higher level, which generalizes the results obtained by individual recognition systems. In the most widespread version of such a scheme, the voting procedure is applied. Adjusting the thresholds and parameters of the voting system, errors of type II (as the most critical ones) can be reduced to a minimum at the top level of the analysis.

In addition to the hierarchy of the system being developed, one can take into account the following. By registering physiological signals (ECG, seismic cardiogram, flowmetry data) at a certain point in time, we evaluate the functional state of the CVS at the time of registration. Estimating a state of the same patient at different times, we get a time series of functional state assessments and can detect the start of the transition process from normal to pathology. In addition, the possibility of considering several different data in time for the same patient makes it possible to clarify what is the individual norm for this person, since the norm in such a question is a rather conventional situation, and to build its individual profile. Such data is usually valuable for the creation of cardiac reference systems and the replenishment of databases that are necessary for the construction of expert systems and are, as a rule, a bottleneck for their development and renewal.

There are several different options for implementing a state identification system, both based on machine learning methods (deep neural networks in particular), and other classes of expert systems based on databases and knowledge bases. It is possible to single out at least two large classes containing several subclasses. 
The first embodiment of the pattern recognition system is based on mathematical analysis of physiological signals and consists in the direct processing of the analyzed time series representing them. In such an embodiment, when the time series, e.g. ECG or flowmetry, are fed directly to the input, the advantages lie in the simplicity of preparing such data for neural network analysis: you can begin the recognition almost immediately after reading the indications, paying attention to simple primary processing procedures, such as filtering, noise removal, anti-aliasing, and etc. Disadvantages are the large volumes of data fed to neural networks, which slows down the process of both training and operation. It also significantly reduces the possibility of using such an option in telemedicine, due to the difficulty of transferring such data volumes. If there are significant volumes of experimental data, when a sufficient number of objects of each class can be found, and also there is information about whether the selection of experimental data sampling objects is random, deep learning methods based on the use of multilayer neural networks are successfully used. At present, deep learning methods are actively developing and prove their success in processing time series of various nature, including ECG signals, seismic cardiograms, etc.

The second variant of the implementation of the CVS state identification system, which includes a lot of possibilities for adaptation, consists in analyzing not the time series itself, but some extracted features from these series. In this case, it can be either the calculation of particular characteristics of the internal organization and the structure of the series that allow us to distinguish between time series belonging to different classes or the construction of certain spectral representations that allow us to use the spectral composition or shape of the spectrum for classification or the result of some tertiary analysis. This can be done either as a preliminary step in the preparation for using neural networks for classification, or as an additional step-extractor in a multistage neural network. Accordingly, there are three different approaches to distinguishing characteristics from time series.

A first approach to time series analysis is to calculate the individual numerical parameters describing certain properties of the time series being analyzed. It can be standard deviation, variance, entropy, Hurst exponent, fractal dimension and other parameters [11]. For example, the hypothesis is proved that the state in the norm corresponds to processes with simpler control and, accordingly, long-term memory, and, therefore, with a smaller fractal dimension, while different pathologies correspond to processes with more complex control, that is, processes with a shorter memory, and, hence, with a larger fractal dimension. The conducted researches $[12,5]$ show that the estimation of such indicators for healthy people and for sick people, significantly differs.

The second approach to analyzing time series is to use time-frequency analysis, i.e. the construction of time series decompositions by various systems of functions and the construction of all possible spectra [5]. This includes Fourier analysis, wavelet analysis, etc. In particular, the construction of scalogramms and skeletons of signals, allows us to see the fundamental differences between the state "in norm" and "in pathology" [13].
The third approach to the analysis of time series is based on the use of some calculated signal-specific indicators in the classification of states. For example, for flowmetry, these are such indicators as the systolic-diastolic coefficient, the pulse wave rise index, velocity integrals over time for different phases of the cardiac cycle, etc. They are calculated using special techniques of phase analysis and also differ significantly for states "in norm" and "in pathology" [14].

Also, an approach is possible here, using the partitioning and coding of time series fragments by the letters of some alphabet. The result is a sequence in a given alphabet, each letter (character) of which corresponds to a certain class of fragments. Each time series can thus be associated with a proper representation of the time raw in the form of a sequence of symbols in the corresponding alphabet. When implementing such a linguistic approach, algorithms for processing character sequences are widely used to identify the most characteristic "grammatical constructions". In accordance with this, the classification of time series can be reduced to a comparative analysis of patterns corresponding to different functional states of the CVS [10].

In this version of the system, a different combination of these approaches is possible to refine the results obtained.

\section{Experiment}

Based on a specialized software realizing the raised and developed technique, numerical experiments with real clinical data were carried out. In this section of the article some results (obtained on ECG signals) of the experiment will be presented.

\section{A. Description of the experiment and the initial data}

ECG data used in this work are obtained from the open database "PhysioNet" [15]. This work uses data from 40 healthy people and 40 patients with various diseases. As preprocessing data for a multilayer perceptron and a onedimensional convolutional neural network, the ECG signals with a single coerced frequency of $250 \mathrm{~Hz}$ were divided into segments for 5 seconds (Fig. 2). In order for the data to be used to train a two-dimensional convolutional neural network, the frequency was reduced to $40 \mathrm{~Hz}$ after segmentation, and the ECG data was quantized in steps of $0.1 \mathrm{mV}$, from $-10 \mathrm{mV}$ to $10 \mathrm{mV}$. After these transformations, the resulting ECG segments were recorded as coordinates and stored as matrixes 200 to 200 .

The evaluation of the networks was carried out in 3 stages:

1. At the first stage, data from half of patients and healthy people are extracted. From diseased people data, ECG data of people with the following diseases were selected: coronary heart disease, heart failure, valvular heart disease, arrhythmia. For the healthy people, ECG data was selected from the CEBS database. In accordance with the method of hold-out set, the 
extracted data is divided into the training and test parts (the training and test parts are $60 \%$ and $40 \%$ respectively from the extracted data). After training the network on the training data, the metrics Accuracy (Acc), Sensitivity (Sen), Specificity (Spe) and $F_{1}$-measure [7] are calculated.

2. At the second stage, the data of the remaining sick and healthy people are extracted.

The problem of constructing a single predictive algorithm for heterogeneous data (from different databases or, for example, from different medical institutions) is not trivial, even large companies are not yet able to fully realize this [4]. This also happens because the routine for preparing and converting the collected data into the proper form for analysis is the main part of the work. Therefore, it is necessary to take into account such parameters as portability and universality, when building a neural network. In order to study these characteristics, this step was added to the course of the experiment, for calculating metrics on new data for a neural network, from another database.

For sick patients this is ECG data of people with the following diseases: transient ischemic attack, myocarditis, myocardial infarction. For healthy people this is the ECG data of people from the PTB database. On the extracted data, the metrics Acc, Sen, Spe, $F_{1}$ are calculated.

3. At the third stage, 4 randomly selected contiguous segments, for 5 seconds each, are extracted from ECG data of each person. If one of the segments is identified by the network as an ECG of sick person, then the person is considered to be sick. The error matrix is calculated from the results of the network operation.

After the verification on all the network architectures used in this article, the architecture that shows the best results is selected. On the chosen architecture, the items 1-3 are reexamined using the cross-validation method.
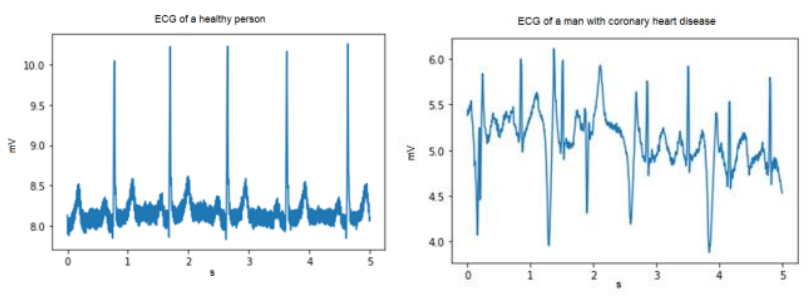

Figure 2. 5 seconds ECG segments of a healthy and sick person

\section{B. Results}

The obtained results show that the practical applicability of convolutional neural networks in this diagnosis task is better than that of a multilayer perceptron (a universal network, which shows stable but not the most effective results). Given that the results for one-dimensional and two-dimensional convolutional neural networks are not much different, and the complexity of data preparation and training for a twodimensional convolutional network is greater than for a one- dimensional one, a one-dimensional convolutional neural network (see Table I) was chosen at the stage of crossvalidation. The results of cross-validation with 10 blocks for the best network option are presented in Table II.

TABLE I. STRUCTURE OF A ONE-DIMENSIONAL CONVOLUTIONAL NEURAL NETWORK

\begin{tabular}{|c|c|c|c|c|c|}
\hline $\begin{array}{c}\text { Lay } \\
\text { er }\end{array}$ & Layer type & $\begin{array}{c}\text { Output } \\
\text { dimension }\end{array}$ & $\begin{array}{c}\text { Dimension } \\
\text { of the } \\
\text { kernel }\end{array}$ & $\begin{array}{c}\text { Number } \\
\text { of filters }\end{array}$ & $\begin{array}{c}\text { Step of } \\
\text { convoluti } \\
\text { on }\end{array}$ \\
\hline 0 & Input & $1250 \times 1$ & - & - & - \\
\hline 1 & $\begin{array}{c}\text { Convolu- } \\
\text { tional }\end{array}$ & $1248 \times 20$ & 3 & 20 & 1 \\
\hline 2 & $\begin{array}{c}\text { Sub- } \\
\text { sampling }\end{array}$ & $624 \times 20$ & 2 & - & - \\
\hline 3 & $\begin{array}{c}\text { Fully } \\
\text { connected }\end{array}$ & 500 & - & - & - \\
\hline 4 & Output & 2 & - & - & - \\
\hline
\end{tabular}

TABLE II. RESULTS OF A ONE-DIMENSIONAL CONVOLUTIONAL NEURAL NETWORK

\begin{tabular}{|c|c|c|c|c|}
\hline Metrics & Acc & Sen & Spe & $\boldsymbol{F}_{\boldsymbol{1}}$ \\
\hline $\begin{array}{c}\text { Training } \\
\text { sample }\end{array}$ & 0.983 & 0.976 & 1 & 0.988 \\
\hline Test sample & 0.974 & 0.965 & 0.997 & 0.981 \\
\hline $\begin{array}{c}\text { Data from } \\
\text { another } \\
\text { base }\end{array}$ & 0.881 & 0.962 & 0.896 & 0.928 \\
\hline $\begin{array}{c}\text { Training } \\
\text { sample } \\
\text { (cross- } \\
\text { validation) }\end{array}$ & 0.984 & 0.978 & 0.998 & 0.988 \\
\hline $\begin{array}{c}\text { Test sample } \\
\text { (cross- } \\
\text { validation) }\end{array}$ & 0.974 & 0.970 & 0.992 & 0.981 \\
\hline
\end{tabular}

\section{Conclusion}

An approach using the methods of machine learning, namely - neural networks, has proved its effectiveness in problems of classification of the patient cardiovascular system state. As a result of all the manipulations described, a branched hierarchy of algorithmic and mathematical models arises. Thus, the generalized model for the mathematical processing of the CVS data by machine learning is a hierarchy at the lower level of which are the structural elements of the time series being analyzed; successive ascent of the hierarchy is the process of sequential identification of patterns of different types; and at the upper level of the multi-level model there is a final decision rule that expresses the end result of the identification system with the aim of supporting decisionmaking. 


\section{References}

[1] N. P. Zaremba, Yu. K. Shlyk, D. V. Krinochkin, "System analysis of solving cardiological tasks. Statement a research task," Proceedings in Cybernetics, 2006, vol. 5, pp. 95-98.

[2] M. Prado, L. Roa, J. Reina-Tosina, "Virtual Center for Renal Support: Technological Approach to Patient Physiological Image," IEEE Transaction on biomedical engineering, 2002, vol. 49, № 12, pp. 14201430.

[3] V.M. Nikitin, V.V. Lomakin, D.A. Anohin, I.K. Kaydalova, I.I. Ivanov, "Information Decision Support System Of Semistructured Problems In Cardiology,” Scientific Statements, 2010, № 19(90), issue 16/1, pp. 112-119.

[4] A. Rajkomar, E. Oren, J. Dean, et al, "Scalable and accurate deep learning with electronic health records," npj Digital Medicine, 2018, article number: 18, DOI: 10.1038/s41746-018-0029-1.

[5] N.S. Konnova, M.A. Basarab, D.A. Basarab, "Digital signal processing in Doppler flowmetry. Methods and algorithms," Monograph, Lambert Academic Publishing, 2018, 233 p.

[6] V.M. Achildiev, V.A. Soldatenkov, N.A. Bedro, Yu.K. Gruzevich, Yu.N. Evseeva, A.D. Levkovich, M.A. Basarab, N.S. Konnova, "Cardioseismometer unit based on micromechanical sensors," Proceedings of 25th Anniversary Saint Petersburg International Conference on Integrated Navigation Systems, 2018, pp. 272-281.

[7] N.S. Konnova, M.A. Basarab, "Cardiovascular states identification using machine learning techniques based on artificial neural networks," Clinical Hemorheology and Microcirculation, IOS Press, in press.

[8] J.H. Tan, Y. Hagiwara, W. Pang, I. Lim, S.L. Oh, M. Adam, R.S. Tan, M. Chen, U.R. Acharya, "Application of stacked convolutional and long short-term memory network for accurate identification of CAD ECG signals," Computers in Biology and Medicine, 2018, vol. 94, pp. 19-26.

[9] S.M. Jadhav, S.L. Nalbalwar, A.A. Ghatol, "Artificial Neural Network Models based Cardiac Arrhythmia Disease Diagnosis from ECG signal Data," International Journal of Computer Applications, 2012, vol. 44, issue 15 , pp. 8-13.

[10] V.M. Uspensky, "Informacionnaya funkciya serdca teoriya i praktika diagnostiki zabolevanij vnutrennih organov metodom informacionnogo analiza ehlektrokardiosignalov," Moscow: PLANETA, 2016, 296 p.

[11] N.S. Konnova, M.A. Basarab, "Digital signal processing of blood flow volume velocity Doppler sensor based on nonlinear dynamics methods," Proceedings of the 10th International Conference "Computer data analysis and modeling: theoretical and applied stochastics," 2013, vol. 2, pp. 114-117.

[12] M.A. Basarab, N.S. Konnova, D.A. Basarab, D.D. Matsievsky, "Analysis of fluctuating blood flow data using the methods of nonlinear dynamics and Allan variance," Proceedings of the 18th Conference of the European Society for Clinical Hemorheology and Microcirculation, Lisbon, Portugal, 2016, p. 48.

[13] M.A. Basarab, D.A. Basarab, N.S. Konnova, D.D. Matsievsky, V.A. Matveev, "Analysis of chaotic and noise processes in a fluctuating blood flow using the Allan variance technique," Clinical Hemorheology and Microcirculation, 2016, vol. 64, issue 4, pp. 921-930.

[14] N.S. Konnova, M.A. Basarab, D.A. Basarab, D.D. Matsievsky, "Methodology of qualitative and quantitative analysis of complex curves of coronary blood flow," Physiology of blood circulation: VI AllRussian with the international participation of the school-conference, 2016, pp. 72-73.

[15] A.L. Goldberger, L.A.N. Amaral, L. Glass, J.M. Hausdorff, P.C. Ivanov, R.G. Mark, J.E. Mietus, G.B. Moody, C.K. Peng, H.E. Stanley, "Physiobank, physiotoolkit, and physionet: components of a new research resource for complex physiologic signals," Circulation, 2000, vol. 101, issue 23, pp. E215-E220.
About Author (s):

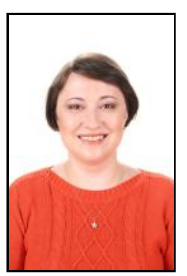

Konnova Natalia Sergeevna, Ph.D., Associate Professor, Bauman Moscow State Technical University. Specializes in the areas of machine learning (artificial intelligence, game theory and operations research) and digital signal processing.

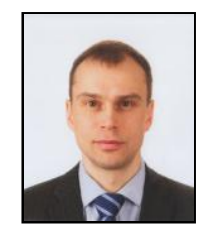

Basarab Mikhail Alekseevich, Dr. Sci. (Phys.-Math), Leading Researcher, Bauman Moscow State Technical University. Scientific interests: applied mathematics, computer science, radiophysics, digital signal processing. 